\title{
Metal-Free Oxidative Dimerization of Dithiocarbamates: Direct Access to 3,5-Bis-mercapto-1,2,4-thiadiazoles
}

\author{
Azim Ziyaei Halimehjani *a \\ Yazdanbakhsh Lotfi Nosooda \\ Shaghayegh Didaran ${ }^{a}$ \\ Fezzeh Aryanasab ${ }^{b}$ \\ ${ }^{a}$ Faculty of Chemistry, Kharazmi University, 49 Mofateh St. \\ PO Box 15719-14911, Tehran, Iran \\ ziyaei@khu.ac.ir \\ ${ }^{\mathrm{b}}$ Department of Chemistry and Petrochemical Engineering, \\ Standard Research Institute (SRI), 31745-139, Karaj, Iran
}

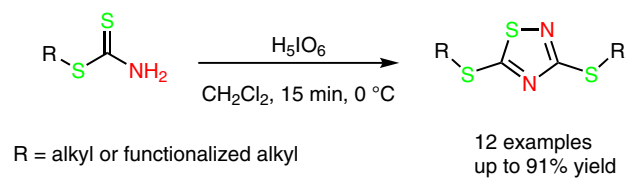

up to $91 \%$ yield

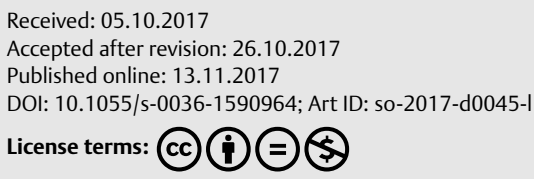

Abstract A facile and efficient protocol for the synthesis of 3,5-bismercapto-1,2,4-thiadiazoles by the oxidative dimerization of S-alkyl dithiocarbamates using periodic acid as an inexpensive and commercially available oxidant is reported. High to excellent yields and short reaction times are the main advantages of this procedure.

Key words 3,5-bismercaptoorganyl-1,2,4-thiadiazoles, dithiocarbamate, oxidative dimerization, hypervalent iodine, transition-metal-free

1,2,4-Thiadiazoles are important heterocyclic compounds due to their widespread use as pesticides, fungicides, herbicides, dyes, and corrosion inhibitors. ${ }^{1}$ Compounds containing the substituted 1,2,4-thiadiazole motif also possess a wide range of biological activities, including antibacterial, ${ }^{2}$ antitumor, ${ }^{3}$ anticonvulsant, ${ }^{4}$ antibiotic, ${ }^{5}$ anti-inflammatory, ${ }^{6}$ and antidiabetic properties. ${ }^{7}$ Furthermore, 1,2,4-thiadiazoles are pharmacophores in the design of novel enzyme inhibitors for targeting the cysteine residues of proteins via disulfide bond formation. ${ }^{8}$

Various methods have been reported for the synthesis of 1,2,4-thiadiazole derivatives, including oxidative dimerization of thioamides and imidoyl thioureas, reaction of $\mathrm{N}$-sulfenylamidines with isothiocyanates, reaction of amidines and amidoximes with carbon disulfide, reaction of thioamidates with chloramine, 1,2,4-thiadiazole ring modifications, ring transformations of oxadiazoles and isoxazoles in the presence of isothiocyanates, rearrangement of dithiazolidines, oxidation of thioacylamidine derivatives, and 1,3-dipolar cycloaddition reactions of nitrile sulfides with nitriles. ${ }^{9}$ Among these methods, the oxidative dimerization of thioamides and intramolecular oxidative S-N bond-formation reactions have been extensively investigat- ed. For this purpose, various oxidants in the presence of a transition metal or under transition metal-free conditions have been developed. ${ }^{10}$ Recently, Yoshimura and co-workers reported the synthesis of 1,2,4-thiadiazoles via oxidative dimerization of carbothioamides using oxone as an inexpensive and eco-friendly oxidant (Scheme 1, Eq. 1). ${ }^{11} \mathrm{Ad}-$ ditionally, Muthusubramanian and co-workers reported the synthesis of 3-substituted-5-amino-1,2,4-thiadiazoles via intramolecular oxidative $\mathrm{S}-\mathrm{N}$ bond formation in the presence of hypervalent iodine(III) (Scheme 1, Eq. 2); ${ }^{12}$ while Kim and co-workers reported a copper catalyzed approach to 3-substituted-5-amino-1,2,4-thiadiazoles from amidine hydrochlorides and isothiocyanates (Scheme 1, Eq. 3). ${ }^{13}$ In a continuation of our interest in the development of dithiocarbamate chemistry, ${ }^{14}$ we report herein a direct route for the synthesis of 3,5-bis-mercapto-1,2,4-thiadiazoles via the simple oxidative dimerization of dithiocarbamates using periodic acid $\left(\mathrm{H}_{5} \mathrm{IO}_{6}\right)$ as oxidant (Scheme 1, Eq. 4).

Initially, the model oxidative dimerization of benzyl carbamodithioate 1a was investigated using an equimolar amount of $\mathrm{H}_{5} \mathrm{IO}_{6}$ in various organic solvents (Table 1, entries 1-6) for $1 \mathrm{~h}$ at room temperature. The best result was observed in $\mathrm{CH}_{2} \mathrm{Cl}_{2}$ and the corresponding 1,2,4-thiadiazole 2a was obtained in 55\% isolated yield (entry 3 ). Additionally, the corresponding benzyl thiocyanate was also observed in the reaction mixtures (15-35\%). After optimization of the solvent, other oxidants including $\mathrm{NaIO}_{4}, \mathrm{KMnO}_{4}, \mathrm{CuCl}_{2}$, and $\mathrm{K}_{2} \mathrm{Cr}_{2} \mathrm{O}_{7}$ in $\mathrm{CH}_{2} \mathrm{Cl}_{2}$ were examined; but lower yields of 1,2,4-thiadiazole 2a were obtained in comparison to $\mathrm{H}_{5} \mathrm{IO}_{6}$ (entries 7-10). Next, we focused on preventing the formation of side-products. Upon decreasing the reaction time from $1 \mathrm{~h}$ to $15 \mathrm{~min}$, the yield of thiadiazole $\mathbf{2 a}$ increased from $55 \%$ to $65 \%$ (entries 11-13); however, further decreasing the reaction time to $10 \mathrm{~min}$ resulted in a lower yield (entry 14). Furthermore, whereas raising the reaction temperature from room temperature to $50{ }^{\circ} \mathrm{C}$ decreased the 
1) Previous work (Yoshimura and co-workers) ${ }^{11}$

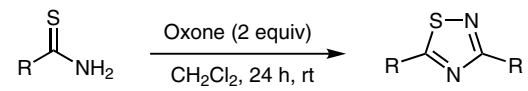

2) Previous work (Muthusubramanian and co-workers) ${ }^{12}$

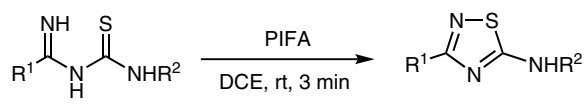

3) Previous work (Kim and co-workers) ${ }^{13}$

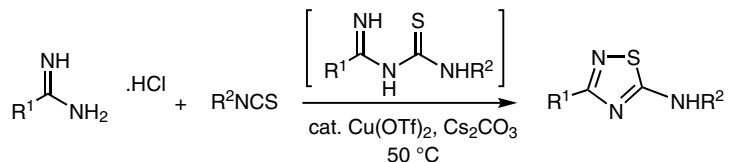

4) This work

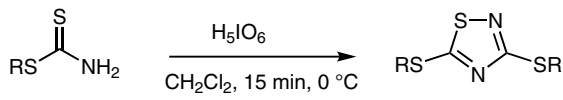

Scheme 1 Synthetic strategies using oxidative dimerization and intramolecular $\mathrm{N}-\mathrm{S}$ bond formation

yield to $40 \%$ (entry 16 ), lowering the reaction temperature to $0{ }^{\circ} \mathrm{C}$ improved the yield to $85 \%$ (entry 15 ). Under these conditions, no benzyl thiocyanate was observed in the reaction mixture. Finally, lower yields were obtained using 0.5 or 2 equivalents of periodic acid (entries 17 and 18). Thus, the oxidative dimerization of dithiocarbamates using an equimolar amount of $\mathrm{H}_{5} \mathrm{IO}_{6}$ in $\mathrm{CH}_{2} \mathrm{Cl}_{2}$ at $0{ }^{\circ} \mathrm{C}$ for 15 min was considered as the optimal reaction conditions for further derivatization.

After optimization of the reaction conditions, the generality of this protocol was examined using various $S$-alkyl dithiocarbamates (Table 2). ${ }^{16}$ The $S$-alkyl dithiocarbamates were prepared according to the reported method via a onepot, three-component reaction between ammonia, $\mathrm{CS}_{2}$, and an electrophile (alkyl halides or $\alpha, \beta$-unsaturated carbonyl compounds). ${ }^{15}$ Various $S$-alkyl and ester containing dithiocarbamates were applied successfully in this oxidative dimerization protocol and the corresponding 3,5-bis-mercapto-1,2,4-thiadiazoles were obtained in high to excellent yields (80-95\%). It is notable that no oxidation was observed at the sulfur in the alkylsulfanyl chains.
Table 1 Optimization of the Reaction Conditions for the Preparation of 2 a from 1 a
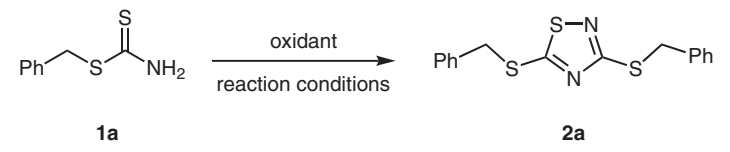

\begin{tabular}{llllll}
\hline Entry & Solvent & Oxidant (equiv) & $\begin{array}{l}\text { Time } \\
\text { (min) }\end{array}$ & $T\left({ }^{\circ} \mathrm{C}\right)$ & Yield (\%) ${ }^{\mathrm{a}, \mathrm{b}}$ \\
\hline 1 & $\mathrm{MeOH}$ & $\mathrm{H}_{5} \mathrm{IO}_{6}(1)$ & 60 & 25 & 30 \\
2 & $\mathrm{EtOH}$ & $\mathrm{H}_{5} \mathrm{IO}_{6}(1)$ & 60 & 25 & 27 \\
3 & $\mathrm{CH}_{2} \mathrm{Cl}_{2}$ & $\mathrm{H}_{5} \mathrm{IO}_{6}(1)$ & 60 & 25 & 55 \\
4 & $\mathrm{THF}$ & $\mathrm{H}_{5} \mathrm{IO}_{6}(1)$ & 60 & 25 & 35 \\
5 & $\mathrm{DMF}$ & $\mathrm{H}_{5} \mathrm{IO}_{6}(1)$ & 60 & 25 & 38 \\
6 & $n-h e x a n e$ & $\mathrm{H}_{5} \mathrm{IO}_{6}(1)$ & 60 & 25 & 20 \\
7 & $\mathrm{CH}_{2} \mathrm{Cl}_{2}$ & $\mathrm{NaIO}_{4}(1)$ & 60 & 25 & 41 \\
8 & $\mathrm{CH}_{2} \mathrm{Cl}_{2}$ & $\mathrm{KMnO}_{4}(1)$ & 60 & 25 & 49 \\
9 & $\mathrm{CH}_{2} \mathrm{Cl}_{2}$ & $\mathrm{CuCl}_{2}(1)$ & 60 & 25 & 34 \\
10 & $\mathrm{CH}_{2} \mathrm{Cl}_{2}$ & $\mathrm{~K}_{2} \mathrm{Cr}_{2} \mathrm{O}_{7}(1)$ & 60 & 25 & 35 \\
11 & $\mathrm{CH}_{2} \mathrm{Cl}_{2}$ & $\mathrm{H}_{5} \mathrm{IO}_{6}(1)$ & 120 & 25 & 50 \\
12 & $\mathrm{CH}_{2} \mathrm{Cl}_{2}$ & $\mathrm{H}_{5} \mathrm{IO}_{6}(1)$ & 30 & 25 & 53 \\
13 & $\mathrm{CH}_{2} \mathrm{Cl}_{2}$ & $\mathrm{H}_{5} \mathrm{IO}_{6}(1)$ & 15 & 25 & 65 \\
14 & $\mathrm{CH}_{2} \mathrm{Cl}_{2}$ & $\mathrm{H}_{5} \mathrm{IO}_{6}(1)$ & 10 & 25 & 45 \\
$\mathbf{1 5}$ & $\mathrm{CH}_{2} \mathrm{Cl}_{2}$ & $\mathrm{H}_{5} \mathrm{IO}_{6}(\mathbf{1})$ & $\mathbf{1 5}$ & $\mathbf{0}$ & $\mathbf{8 5}$ \\
16 & $\mathrm{CH}_{2} \mathrm{Cl}_{2}$ & $\mathrm{H}_{5} \mathrm{IO}_{6}(1)$ & 15 & 50 & 40 \\
17 & $\mathrm{CH}_{2} \mathrm{Cl}_{2}$ & $\mathrm{H}_{5} \mathrm{IO}_{6}(2)$ & 15 & 0 & 60 \\
18 & $\mathrm{CH}_{2} \mathrm{Cl}_{2}$ & $\mathrm{H}_{5} \mathrm{IO}_{6}(0.5)$ & 15 & 0 & 45 \\
\hline
\end{tabular}

a Isolated yield.

${ }^{b}$ Reaction conditions: dithiocarbamate 1 a $(0.5 \mathrm{mmol})$ and solvent $(2 \mathrm{~mL})$.

The possibility for the cross-oxidative dimerization of dithiocarbamates was investigated using $S$-alkyl dithiocarbamates $\mathbf{1 1}$ and 1e (Scheme 2). The products were separated by preparative TLC. Characterization of these products confirmed that two self-dimerization products, $\mathbf{2 1}$ and $\mathbf{2 e}$, were obtained as major components and cross-dimerization compounds, $\mathbf{3 a}$ and $\mathbf{3 b}$, were isolated as minor products.

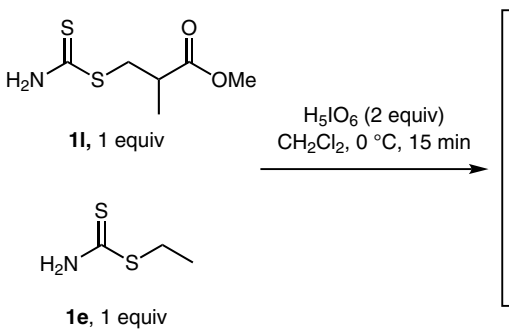

1e, 1 equiv

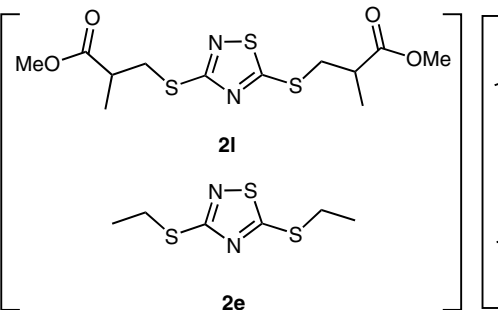

major products self-dimerization products

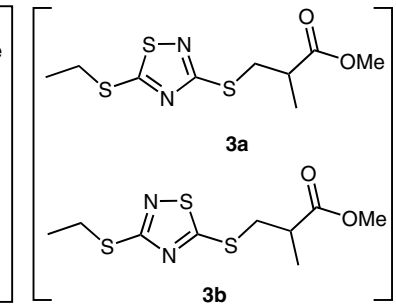

minor products cross-dimerization products

Scheme 2 Cross-oxidative dimerization of $\mathbf{1 l}$ and $\mathbf{1 e}$ 
<smiles>COP(=O)(O)(O)(O)OCCCCSC(N)=S</smiles><smiles></smiles>

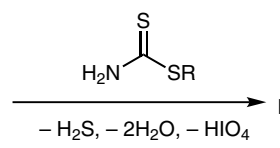

$\rightarrow \mathrm{RS}$

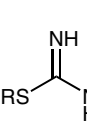

B<smiles>[B]C1=NCCS(C)([Si](C)(O)O)C(P)=N1</smiles>

C $\downarrow-3 \mathrm{H}_{2} \mathrm{O},-\mathrm{HIO}_{3}$<smiles>[Sb]c1nsc([SbH])n1</smiles>

Scheme 3 Proposed mechanism for oxidative dimerization of dithiocarbamates

A proposed mechanism for the oxidative dimerization of dithiocarbmates is given in Scheme 3. Initially, reaction of dithiocarbamate $\mathbf{1}$ with periodic acid affords intermediate $\mathbf{A}$, which undergoes further reaction with dithiocarbamate 1 to provide intermediate $\mathbf{B}$ after elimination of $\mathrm{H}_{2} \mathrm{~S}$, $2 \mathrm{H}_{2} \mathrm{O}$, and $\mathrm{HIO}_{4}$. Then, intramolecular $\mathrm{N}-\mathrm{S}$ bond formation in the presence of periodic acid followed by loss of $\mathrm{HIO}_{3}$ and three equivalents of water gives the corresponding $1,2,4-$ thiadiazole 2.
The structures of all products were confirmed by IR, ${ }^{1} \mathrm{H}$ and ${ }^{13} \mathrm{C}$ NMR spectroscopy, and CHN analysis. The protons of the two alkyl groups of the $S$-alkyl moieties were observed at similar chemical shifts in the ${ }^{1} \mathrm{H}$ NMR spectra. The ${ }^{13} \mathrm{C}$ NMR spectra showed all carbons of both alkyl groups distinctively and the two carbons of the 1,2,4-thiadiazole ring appeared at $170-171$ and $187-188 \mathrm{ppm}$, for all compounds. Compounds $\mathbf{2} \mathbf{g}$ and $\mathbf{2 l}$ were obtained as mixtures of three stereoisomers (including a meso-compound), which were not separated.

Table 2 Diversity in the Oxidative Dimerization Reaction of Dithiocarbamates $\mathbf{1}^{\mathrm{a}}$

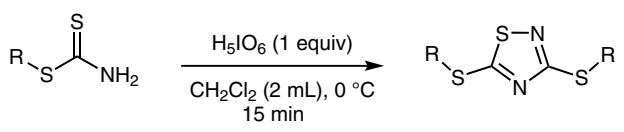

12

Entry Yield (\%)


Table 2 (continued)

\begin{tabular}{|c|c|c|c|}
\hline Entry & Dithiocarbamate 1 & Product $\mathbf{2}$ & Yield $(\%)^{\mathrm{b}}$ \\
\hline 6 & 1f & & 80 \\
\hline 7 & $1 \mathrm{~g}$ & & 86 \\
\hline 8 & & & 83 \\
\hline 9 & & & 91 \\
\hline 10 & & & 83 \\
\hline 11 & & & 80 \\
\hline 12 & 익 & & 82 \\
\hline
\end{tabular}

a Reaction conditions: Dithiocarbamate $1(0.5 \mathrm{mmol}), \mathrm{H}_{5} \mathrm{IO}_{6}(0.5 \mathrm{mmol}), \mathrm{CH}_{2} \mathrm{Cl}_{2}(2 \mathrm{~mL}), 0{ }^{\circ} \mathrm{C}, 15 \mathrm{~min}$.

${ }^{\mathrm{b}}$ Isolated yield.

In conclusion, we have developed a facile and efficient approach for the synthesis of 3,5-bis-mercapto-1,2,4-thiadiazoles by the oxidative dimerization of $S$-alkyl dithiocarbamates in the presence of periodic acid. High to excellent yields and short reaction times are the main advantages of this protocol.

\section{Acknowledgment}

We are grateful to the Faculty of Chemistry of Kharazmi University for supporting this work.

\section{Supporting Information}

Supporting information for this article is available online at https://doi.org/10.1055/s-0036-1590964.

\section{References and Notes}

(1) (a) Martinez, A.; Fernandez, E.; Castro, A.; Conde, S.; RodriguezFranco, I.; Banos, J. E.; Badia, A. Eur. J. Med. Chem. 2000, 35, 913.

(b) Lanzafame, A.; Christopoulos, A. J. Pharmacol. Exp. Ther.
2004, 308, 830. (c) Wilkins, D. Comprehensive Heterocyclic Chemistry III; Katritzky, A. R.; Ramsden, C. A.; Scriven, E. F. V.; Taylor, R. J. K.; Zhdankin, V. V., Eds.; Elsevier: Oxford, UK, 2008, 487.

(2) (a) Yamanaka, T.; Ohki, H.; Ohgaki, M.; Okuda, S.; Toda, A.; Kawabata, K.; Inoue, S.; Misumi, K.; Itoh, K.; Satoh, K. U. S. Patent US2005004094 A1, 2005. (b) Camoutsis, C.; Geronikaki, A.; Ciric, A.; Soković, M.; Zoumpoulakis, P.; Zervou, M. Chem. Pharm. Bull. 2010, 58, 160. (c) Onkol, T.; Doğruer, D. S.; Uzun, L.; Adak, S.; Ozkan, S.; Sahin, M. F. J. Enzyme Inhib. Med. Chem. 2008, 23, 277.

(3) Kumar, D.; Kumar, N. M.; Chang, K. H.; Gupta, R.; Shah, K. Bioorg. Med. Chem. Lett. 2011, 21, 5897.

(4) Foroumadi, A.; Tabatabai, S. A.; Gitinezhad, G.; Zarrindast, M. R.; Shafiee, A. Pharm. Pharmacol. Commun. 2000, 6, 31.

(5) (a) Hara, R.; Sakamoto, K.; Hisamichi, H.; Nagano, N. J. Antibiot. 1996, 49, 1162. (b) Ishikawa, T.; Iizawa, Y.; Okonogi, K.; Miyake, A. J. Antibiot. 2000, 53, 1053.

(6) Boschelli, D. H.; Connor, D. T. U. S. Patent US2005114958 A, 2005.

(7) Johnstone, C.; Mckerrecher, D.; Pike, K. G.; Waring, M. J. PCT Int. Appl. WO2005121110A1, 2005.

(8) Tam, T. F.; Leung-Toung, R.; Li, W.; Spino, M.; Karimian, K. Mini-Rev. Med. Chem. 2005, 5, 367. 
(9) (a) Wilkins, D. J.; Bradley, P. A. Product Class 10: 1,2,4-Thiadiazoles, In Science of Synthesis: Houben-Weyl Methods of Molecular Transformations, 5th ed.; Thieme: Stuttgart, Germany, 2004. (b) Wehn, P. M.; Harrington, P. E.; Eksterowicz, J. E. Org. Lett. 2009, 11, 5666. (c) Frija, L. M. T.; Pombeiro, A. J. L.; Kopylovich, M. N. Eur. J. Org. Chem. 2017, 2670.

(10) (a) Castro, A.; Castano, T.; Encinas, A.; Porcal, W.; Gil, C. Bioorg. Med. Chem. 2006, 14, 1644. (b) Park, J. Y.; Ryu, I. A.; Park, J. H.; Chan, D.; Gong, Y. D. Synthesis 2009, 913. (c) Patil, P. C.; Bhalerao, D. S.; Dangate, P. S.; Akamanchi, K. G. Tetrahedron Lett. 2009, 50, 5820. (d) Mamaeva, E. A.; Bakibaev, A. A. Tetrahedron 2003, 59, 7521. (e) Cheng, D. P.; Chen, Z. C. Synth. Commun. 2002, 32, 2155. (f) Thorat, P. B.; Bhong, B. Y.; Karade, N. N. Synlett 2013, 2061. (g) Mayhoub, A. S.; Kiselev, E.; Cushman, M. Tetrahedron Lett. 2011, 52, 4941.

(11) Yoshimura, A.; Todora, A. D.; Kastern, B. J.; Koski, S. R.; Zhdankin, V. V. Eur.J. Org. Chem. 2014, 5149.

(12) Mariappan, A.; Ragaguru, K.; Chola, N. M.; Muthusubramanian, S.; Bhuvanesh, N. J. Org. Chem. 2016, 81, 6573.

(13) Kim, H. Y.; Kwak, S. H.; Lee, G. H.; Gong, Y. D. Tetrahedron 2014, $70,8737$.

(14) (a) Ziyaei, Halimehjani. A.; Hajiloo, Shayegan. M.; Hashemi, M. M.; Notash, B. Org. Lett. 2012, 14, 3838. (b) Ziyaei, Halimehjani. A.; Torabi, S.; Amani, V.; Notash, B.; Saidi, M. R. Polyhedron 2015, 102, 643. (c) Azizi, N.; Aryanasab, F.; Torkian, L.; Ziyaei, A.; Saidi, M. R. J. Org. Chem. 2006, 71, 3634. (d) Ziyaei, Halimehjani. A.; Hosseinkhany, S. Synthesis 2015, 47, 3147. (e) Ziyaei, Halimehjani. A.; Khoshdoun, M. J. Org. Chem. 2016, 81, 5699. (f) Schlüter, T.; Ziyaei, Halimehjani. A.; Wachtendorf, D.; Schmidtmann, M.; Martens, J. ACS Comb. Sci. 2016, 18, 456. (g) Ziyaei, Halimehjani. A.; Marjani, K.; Ashouri, A. Tetrahedron Lett. 2012, 53, 3490. (h) Ziyaei, Halimehjani. A.; Martens, J.; Schlüter, T. Tetrahedron 2016, 72, 3958.

(15) (a) Ziyaei, Halimehjani. A.; Hasani, L.; Alaei, M. A.; Saidi, M. R. Tetrahedron Lett. 2016, 57, 883. (b) Ziyaei, Halimehjani. A.; Airamlounezhad, S. J. Heterocycl. Chem. 2014, 51, 1147. (c) Ziyaei, Halimehjani. A.; Alaei, M. A.; Soleymani, Movahed. F.; Jomeh, N.; Saidi, M. R. J. Sulfur Chem. 2016, 37, 529.
(16) Synthesis of 3,5-(Bismercaptoorganyl)-1,2,4-thiadiazoles; General Procedure: To a solution of dithiocarbamate $1(0.5$ $\mathrm{mmol})$ in $\mathrm{CH}_{2} \mathrm{Cl}_{2}(2 \mathrm{~mL})$, periodic acid $(0.5 \mathrm{mmol})$ was added. The resulting mixture was stirred at $0{ }^{\circ} \mathrm{C}$ for 15 minutes. After completion of the reaction, water $(3 \mathrm{~mL})$ was added and the mixture was extracted with $\mathrm{CH}_{2} \mathrm{Cl}_{2}(3 \times 3 \mathrm{~mL})$. The combined organic extracts were dried over $\mathrm{Na}_{2} \mathrm{SO}_{4}$, filtered and evaporated under reduced pressure. The desired product 2 was purified by column chromatography (silica gel; ethyl acetate/hexane, 1:10).

\section{Characterization Data for Selected Compounds}

3,5-Bis(benzylthio)-1,2,4-thiadiazole (2a): Yield: $70 \mathrm{mg}$ (85\%); yellow oil. IR (KBr): 1424, 1211, 1041, $699 \mathrm{~cm}^{-1}$; ${ }^{1} \mathrm{H}$ NMR (300 $\left.\mathrm{MHz}, \mathrm{CDCl}_{3}\right): \delta=4.47(\mathrm{~s}, 4 \mathrm{H}), 7.27-7.45(\mathrm{~m}, 10 \mathrm{H}) ;{ }^{13} \mathrm{C}$ NMR $(75$ $\left.\mathrm{MHz}, \mathrm{CDCl}_{3}\right): \delta=36.4,38.4,127.4,128.1,128.5,128.8,129.0$, 129.1, 135.0, 136.8, 170.2, 187.2; Anal. Calcd (\%) for $\mathrm{C}_{16} \mathrm{H}_{14} \mathrm{~N}_{2} \mathrm{~S}_{3}$ : C, 58.15; H, 4.27; N, 8.48; Found: C, 58.27; H, 4.17; N, 8.35.

3,5-Bis(isopentylthio)-1,2,4-thiadiazole (2b): Yield: $65 \mathrm{mg}$ (89\%); yellow oil. IR (KBr): 1427, 1209, $1041 \mathrm{~cm}^{-1} ;{ }^{1} \mathrm{H}$ NMR (300 $\left.\mathrm{MHz}, \mathrm{CDCl}_{3}\right): \delta=0.93-0.96(\mathrm{~m}, 12 \mathrm{H}), 1.63-1.76(\mathrm{~m}, 6 \mathrm{H}), 3.19-$ $3.25(\mathrm{~m}, 4 \mathrm{H}) ;{ }^{13} \mathrm{C}$ NMR $\left(75 \mathrm{MHz}, \mathrm{CDCl}_{3}\right): \delta=22.1,22.2,27.4$, 27.5, 30.2, 32.3, 37.5, 38.1, 171.1, 187.9; Anal. Calcd (\%) for $\mathrm{C}_{12} \mathrm{H}_{22} \mathrm{~N}_{2} \mathrm{~S}_{3}$ : C, 49.61; H, 7.63; N, 9.64; Found: C, 49.32; H, 7.55; N, 9.49 .

Dibutyl 3,3'-[(1,2,4-Thiadiazole-3,5-diyl)bis(sulfanediyl)]dipropanoate (2k): Yield: $81 \mathrm{mg}(80 \%)$; yellow oil. IR (KBr): 1735 , $1465,1430,1350,1213,1063 \mathrm{~cm}^{-1} ;{ }^{1} \mathrm{H}$ NMR $\left(300 \mathrm{MHz}, \mathrm{CDCl}_{3}\right)$ : $\delta=0.88-0.93(\mathrm{~m}, 6 \mathrm{H}), 1.31-1.39(\mathrm{~m}, 4 \mathrm{H}), 1.57-1.62(\mathrm{~m}, 4 \mathrm{H})$, 2.78-2.85 (m $4 \mathrm{H}), 3.40-3.50(\mathrm{~m}, 4 \mathrm{H}), 4.07-4.12(\mathrm{~m}, 4 \mathrm{H}) ;{ }^{13} \mathrm{C}$ $\operatorname{NMR}\left(75 \mathrm{MHz}, \mathrm{CDCl}_{3}\right): \delta=13.6,19.0,26.9,29.0,30.5,33.1,33.8$, 34.0, 34.4, 34.7, 64.6, 64.8, 169.9, 171.1, 171.6, 187.2; Anal. Calcd (\%) for $\mathrm{C}_{16} \mathrm{H}_{26} \mathrm{~N}_{2} \mathrm{O}_{4} \mathrm{~S}_{3}$ : C, 47.26; $\mathrm{H}, 6.45 ; \mathrm{N}, 6.89$; Found: C, 47.17; H, 6.32; N, 7.05 